\title{
XMM-Newton observations of OY Car III: OM light curve modelling, X-ray timing and spectral studies
}

\author{
P. Hakala ${ }^{1}$ and G. Ramsay ${ }^{2}$ \\ 1 Observatory, PO Box 14, 00014 University of Helsinki, Finland \\ ${ }^{2}$ Mullard Space Science Laboratory, University College London, Holmbury St Mary, Dorking, Surrey, RH5 6NT, UK
}

Received 8 July 2003 / Accepted 3 December 2003

\begin{abstract}
We revisit the XMM-Newton observations of the dwarf nova OY Car taken in July 2000 which occured shortly after an outburst. Ramsay et al. (2001a) found a prominent energy dependent modulation at a period of $2240 \mathrm{~s}$ : this modulation was only seen for $\sim 1 / 3$ of the observation duration. In our new analysis, we examine this time interval in greater detail. In addition to the $2240 \mathrm{~s}$ period we find evidence for other periods, the most prominent being near $3500 \mathrm{~s}$. Both these modulations are most likely due to changes in photoelectric absorption over this period: this is supported by phase-resolved spectroscopy. This may indicate the presence of matter above the accretion disc or a presence of a magnetic accretion curtain. In this case the $2240 \mathrm{~s}$ period could represent a spin period of the white dwarf and the $3500 \mathrm{~s}$ period a beat period between the spin and orbital periods. We also model the $B$ band and UV eclipse profiles and light curves using a new technique to map the spatial extent of the accretion disc. As a result we find that whilst the optical emission is dominated by both the emission close to the accretion disc boundary layer and the hot spot where the accretion stream hits the disc, the UV emission is mainly dominated by the inner disc/boundary layer only.
\end{abstract}

Key words. accretion, accretion discs - stars: binaries: eclipsing - stars: individual: OY Car - stars: novae, cataclysmic variables - X-rays: stars

\section{Introduction}

Dwarf novae are interacting stellar binary systems in which material gets transfered from a red dwarf secondary star onto a white dwarf via Roche lobe overflow. In the absence of a strong magnetic field this material forms an accretion disc around the white dwarf. Dwarf novae show outbursts which occur on week to month timescales. For systems which are viewed close to the plane of the binary system, eclipses of the white dwarf by the secondary star are seen every orbital period. These systems are important since they allow a detailed study of the accretion disc as the secondary gradually covers then uncovers the disc.

One such eclipsing dwarf nova is OY Car. It has a binary orbital period of $1.51 \mathrm{~h}$ and has been extensively studied at various wavelengths. Recently, it has been observed at X-ray and optical/UV energies using XMM-Newton. Ramsay et al. (2001a) found that there was a quasi-stable modulation of the $\mathrm{X}$-rays at $\sim 2240 \mathrm{~s}$, which was most prominent at lower energies. They speculated that this was the due to disc material orbiting above the binary orbital plane or due to the spin period of the white dwarf. In the latter scenario, this would represent the first time that the spin period of the white dwarf in a dwarf nova had been detected.

Send offprint requests to: $\mathrm{P}$. Hakala, e-mail: pasi.hakala@astro.helsinki.fi
The eclipse profile shows a sharp drop in X-rays and in the UV band. In X-rays the flux remains at a very low, but significant level after this rapid (20-30 s) drop. In the UV and $B$ bands, the flux decreases at a more gradual rate after the rapid drop since the accretion disc is still visible after the eclipse of the white dwarf. Wheatley \& West (2003) further analysed the XMM-Newton data and found that the X-ray eclipse lasted $30 \pm 3 \mathrm{~s}$ and concluded the X-ray emission originated at the poles of the white dwarf.

In contrast, in this paper we investigate the nature of the $2240 \mathrm{~s}$ modulation in more detail by revisiting the $X M M-N e w t o n$ data. In particular we have carried out phased resolved spectroscopy over this modulation period. Further, we apply a new eclipse mapping technique to model the spatial distribution of the accretion disc using the OM XMM-Newton data.

\section{Observations}

XMM-Newton was launched in December 1999 and has the largest effective area of any imaging X-ray telescope. It has 3 medium spectral resolution CCD type cameras on-board: two EPIC MOS detectors and one EPIC pn detector. Since the EPIC pn detector has the higher sensitivity we only discuss data taken using this detector (Strüder et al. 2001). In addition, it has 

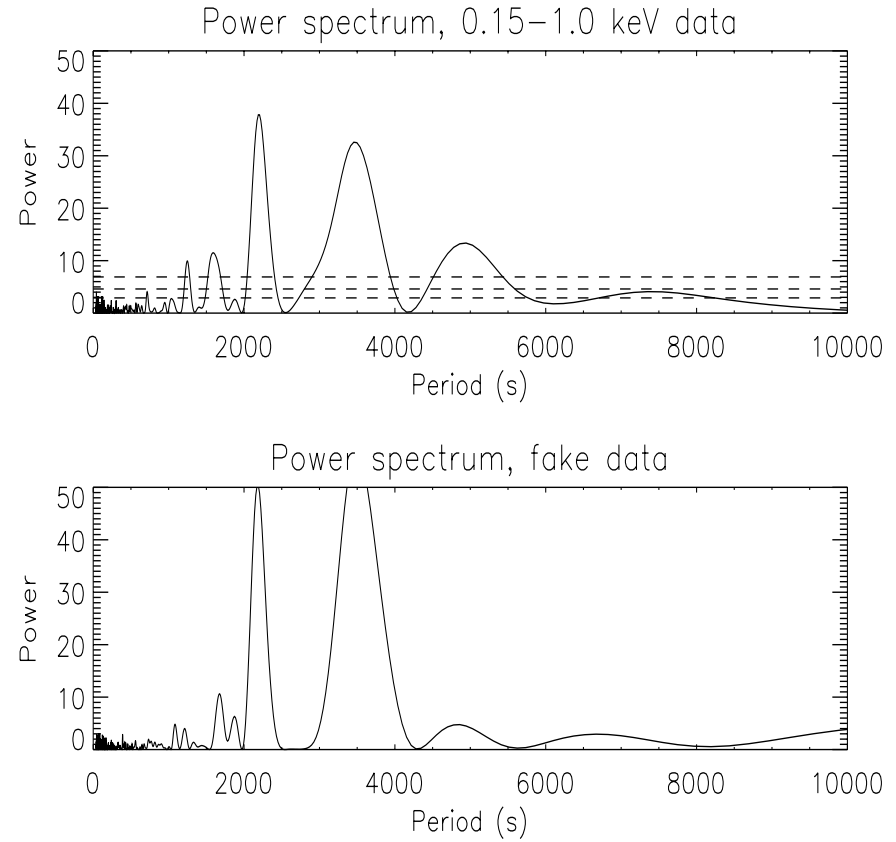

Fig. 1. The 0.15-1.0 keV power spectrum with $95 \%, 99 \%$ and $99.9 \%$ confidence limits (three dashed lines) from our simulations (top) and a power spectrum produced using simulated fake data that contains the two strongest signals (2193 s and $3510 \mathrm{~s}$ ) seen in the power spectrum. The shapes of $2193 \mathrm{~s}$ and $3510 \mathrm{~s}$ pulses are estimated using a simultaneous 2 nd order fourier fit with two periods.

an $0.3 \mathrm{~m}$ optical/UV imaging telescope which enables simultaneous X-ray optical/UV observations (Mason et al. 2001).

OY Car was observed during the performance verification phase of the mission on June 29-30th 2000. The details of the observation are reported in Ramsay et al. (2001a,b). However, the main points to note are that these observations were made some 4 days after an optical outburst. The system had already faded by $V \sim 4$ mag from its peak, but it was still relatively bright in X-rays ( $\sim 1.1 \mathrm{ct} \mathrm{s}^{-1}$ in the EPIC pn). The exposure in the EPIC pn detector was $48 \mathrm{ks}$, it was configured in full frame mode and the medium filter was used. The optical data were obtained in the $U V W 1$ filter (effective wavelength $2910 \AA$ ). The data were processed using the XMM-Newton Science Analysis System v5.3.3. Additional optical data in $B$ band was obtained in August 2000 when it was at a similar brightness to the June observation (Ramsay et al. 2001a).

\section{X-ray light curve analysis}

Ramsay et al. (2001a) found evidence for a quasi-stable X-ray modulation on a period of $\sim 2240 \mathrm{~s}$, which was strongest in soft X-rays. This modulation was observed for around $1 / 3$ of the duration of the observation and it was speculated that this period was related to the spin period of the white dwarf.

We have re-examined the light curves of OY Car: in particular we perform a timing analysis of that portion of the light curve in which the $2240 \mathrm{~s}$ modulation is strongest (HJD 2451725.6548-2451725.8622) in 4 energy bands (0.15$10.0,0.15-1.0,1-4$ and $4-10 \mathrm{keV}$ ). (We removed the time of the eclipse from the light curves.) We show the amplitude

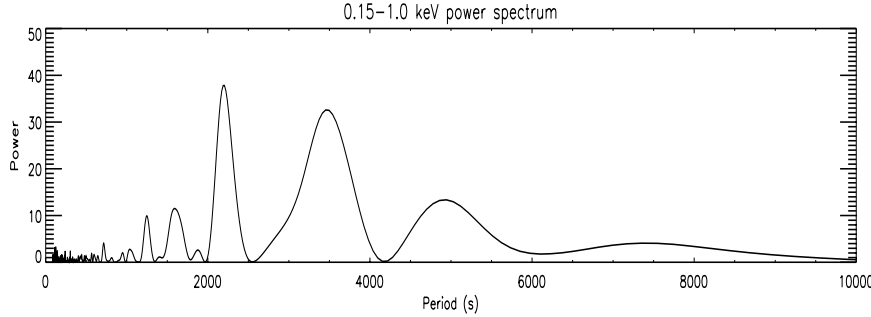

1.0-4.0 keV power spectrum

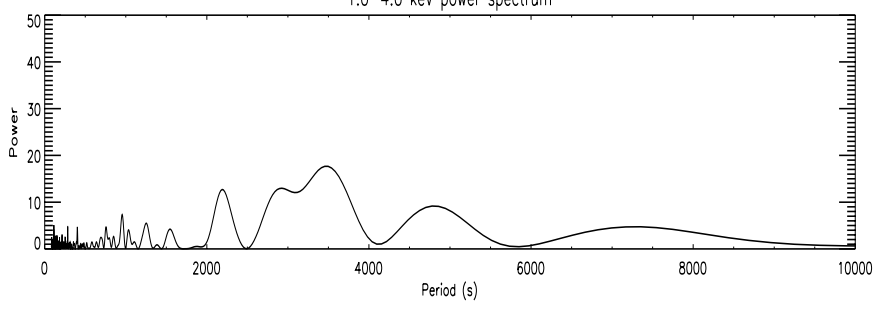

4.0-10.0 keV power spectrum

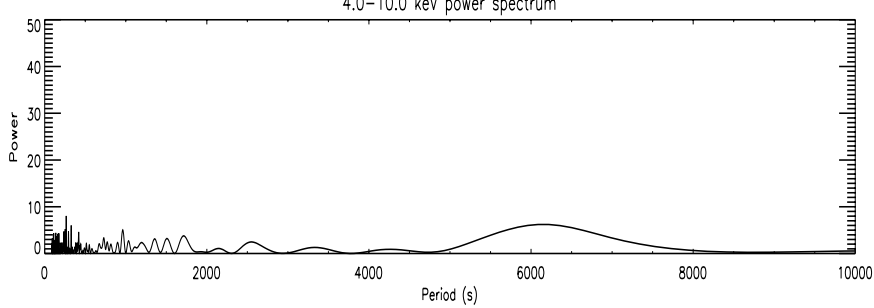

Fig. 2. The power spectra at $0.15-1.0 \mathrm{keV}$ (top), $1.0-4.0 \mathrm{keV}$ (middle) and $4.0-10.0 \mathrm{keV}$ (bottom).

spectrum of the 0.15-1.0 keV light curve in Fig. 1 (top panel). The most prominent peak has a period of $2193 \mathrm{~s}$ : within the errors, this period is consistent with the 2240 s period found by Ramsay et al. (2001a). However, there is a second prominent peak at $3510 \mathrm{~s}$ : this peak is still present if we pre-whiten the data on the $2193 \mathrm{~s}$ period. We performed the same analysis in the 2 more restricted energy bands (Fig. 2): we find that both the $2193 \mathrm{~s}$ and $3510 \mathrm{~s}$ periods are more prominent at softer energies. A peak was seen at $3510 \mathrm{~s}$ in the amplitude spectrum shown in Ramsay et al. (2001a), however, it is more prominent in this new study since we have restricted the analysis to the time interval in which the quasi-stable modulation is seen. In order to demonstrate the prominence of both of these periods, we show the data folded on either of the two periods, after they have been prewhitened using the other period (Fig. 3).

To determine the significance of these two periods we randomly populated the time bins with the observed count rates. We then generated 10000 power density spectra: the 95\%, 99\% and $99.9 \%$ confidence limits from these are shown together with the 0.1-1.0 keV power spectrum in Fig. 1 (top panel). It is clear that both the 2193 and 3510 s periods are highly significant. To determine the errors on these periods we used the Cash statistic (Cash 1979), which implies: $2193_{-35}^{+17}$ and $3510_{-104}^{+25} \mathrm{~s}$. There are three other periods which are significant at $>99.9 \%$ level: $1248_{-15}^{+17} \mathrm{~s}, 1574_{-21}^{+18} \mathrm{~s}$ and $4844_{-104}^{+120} \mathrm{~s}$.

It is possible that some of these less significant periods could be produced as a result of aliasing between the most prominent periods (2193 s and $3510 \mathrm{~s}$ ) and the window function or as a result of these two periods having a non-sinusoidal pulse shape. In order to investigate this further we have 

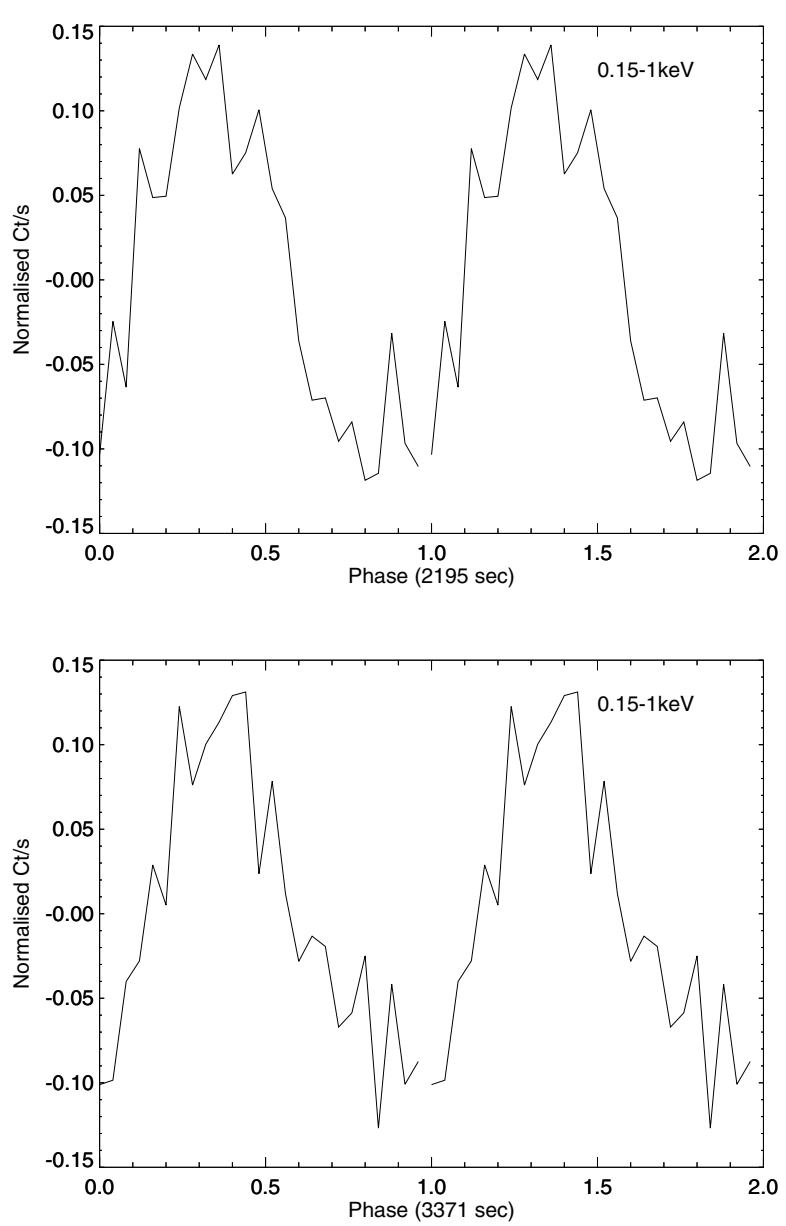

Fig. 3. The $0.15-1.0 \mathrm{keV}$ light curve prewhitened on the $3510 \mathrm{~s}$ period and then folded on the $2193 \mathrm{~s}$ period (top panel), and vice-versa (bottom panel).

simulated a dataset with the same time points as the original data. However, this fake data only contains the two strongest signals $(2193 \mathrm{~s}$ and $3510 \mathrm{~s})$. The shape of these signals is obtained from the real data by using a simultaneous two-period fourier fit, where each of the two pulse shapes are represented with a second order fourier series. As a result we find that at least the $1574 \mathrm{~s}$ period could be produced by the effects mentioned above, Fig. 1 (lower panel).

We discuss the possible origin of all of these periods in Sect. 6.1.

\section{X-ray spectral analysis}

Taking the same time interval that the quasi-stable behaviour is present as in the previous section, we phased events on the 2193 and $3510 \mathrm{~s}$ quasi-stable periods reported in the previous section. Initially we extracted 6 spectra covering different 2193 s phase intervals. In extracting these spectra we used apertures $\sim 30^{\prime \prime}$ in radius centered on OY Car, chosen so that the aperture did not cover more than one CCD. This encompasses $\sim 90$ percent of the integrated PSF (Aschenbach et al. 2000). A background spectrum was extracted from the same CCD on which the source was detected, scaled and subtracted from the source spectra.
Table 1. The results of phase resolved spectral fits folded on the $2193 \mathrm{~s}$ period. The temperature of the cooler $\left(T_{1}\right)$ plasma was fixed at $1.4 \mathrm{keV}$, the hotter $\left(T_{2}\right)$ at $10 \mathrm{keV}$.

\begin{tabular}{llrrr}
\hline \hline Phase & $\begin{array}{l}N_{H} \\
\left(10^{20}\right.\end{array}$ & $\begin{array}{r}\text { Norm } T_{1} \\
\left(\times 10^{-4}\right)\end{array}$ & $\begin{array}{r}\text { Norm } T_{2} \\
\left(\times 10^{-3}\right)\end{array}$ & $\begin{array}{r}\chi_{v}^{2} \\
(\text { d.o.f. })\end{array}$ \\
& $\left.\mathrm{cm}^{-2}\right)$ & & & \\
\hline $0.0-0.17$ & $9.7 \pm 1.3$ & $1.81_{-0.71}^{+0.64}$ & $2.14_{-0.11}^{+0.13}$ & $1.50(62)$ \\
$0.17-0.32$ & $7.5_{-1.1}^{+1.3}$ & $2.34_{-0.73}^{+0.63}$ & $1.80 \pm 0.12$ & $1.60(52)$ \\
$0.32-0.50$ & $5.9_{-0.9}^{+1.0}$ & $2.80_{-0.59}^{+0.71}$ & $1.96_{-0.12}^{+0.11}$ & $1.27(72)$ \\
$0.50-0.60$ & $4.2_{-1.1}^{+1.2}$ & $2.68_{-0.81}^{+0.95}$ & $2.13_{-0.17}^{+0.16}$ & $0.71(42)$ \\
$0.60-0.80$ & $7.6_{-1.1}^{+1.2}$ & $1.98_{-0.70}^{+0.56}$ & $2.10_{-0.11}^{+0.12}$ & $1.18(75)$ \\
$0.80-1.00$ & $10.9_{-1.2}^{+1.4}$ & $2.00_{-0.68}^{+0.60}$ & $1.96_{-0.10}^{+0.12}$ & $1.47(67)$ \\
\hline
\end{tabular}

Since the response of the detectors is not well calibrated at present below $\sim 0.2 \mathrm{keV}$, energies below this were ignored in the following analysis. The response file epn_ff20_sdY9_medium.rmf was used: this response includes single and double pixel events.

Ramsay et al. (2001b) found that they could not well fit the integrated EPIC spectra of OY Car with a single temperature thin plasma model. A multi-temperature model gave a much better fit to the data. In their analysis Ramsay et al. (2001b) concluded that a best fit for the integrated spectrum was obtained with a three temperature plasma model with a partially covering absorber. The multi-temperature nature was supported from line $\mathrm{H} / \mathrm{He}$-like intensity ratios of Iron and Sulphur and also by the various line species seen in the high resolution RGS spectrum.

We fitted each of the 6 phase resolved spectra separately using the X-ray fitting package XSPEC. Each spectrum was binned so that a minimum of 40 counts were present in each spectral bin. An initial analysis showed that a two temperature fit gave much better fits to the data. In these phase resolved spectra adding a third temperature component did not significantly improve the fit. Further, we find that the temperatures for each of thermal components were consistent in each of the 6 phase resolved spectra $\left(k T_{1}=1.4 \mathrm{keV}\right.$ and $\left.k T_{2}=10 \mathrm{keV}\right)$. We therefore fixed the temperatures of the thermal plasma models at these temperatures. In each fit the free parameters were the absorption (we used a simple neutral absorption model) and the normalisation. The metal abundance was fixed at solar.

The resulting fits are shown in tabulated form in Table 1 and in graphical form (along with the light curve folded on the 2193 s period) in Fig. 4. The fits are reasonably good in the phase intervals $0.32-0.80$, but in the remainder it is less so $\left(\chi_{v}^{2} \sim 1.5\right)$. In the case of the phase interval 0.0-0.17 the fit was significantly improved when we fix the metal abundance at 1.5 solar $\left(\chi_{v}^{2}=1.35\right)$. It is clear from Fig. 4 that there is no significant variability in the normalisations of the thermal plasma components. However, there is a variation in the column of the neutral Hydrogen density: it is anti-correlated with the folded intensity light in that it shows a maximum at intensity minimum. We show in Fig. 5 the confidence contour plot in the $\left(N_{\mathrm{H}}, T_{\text {low, norm }}\right)$ plane for the spectra covering the 


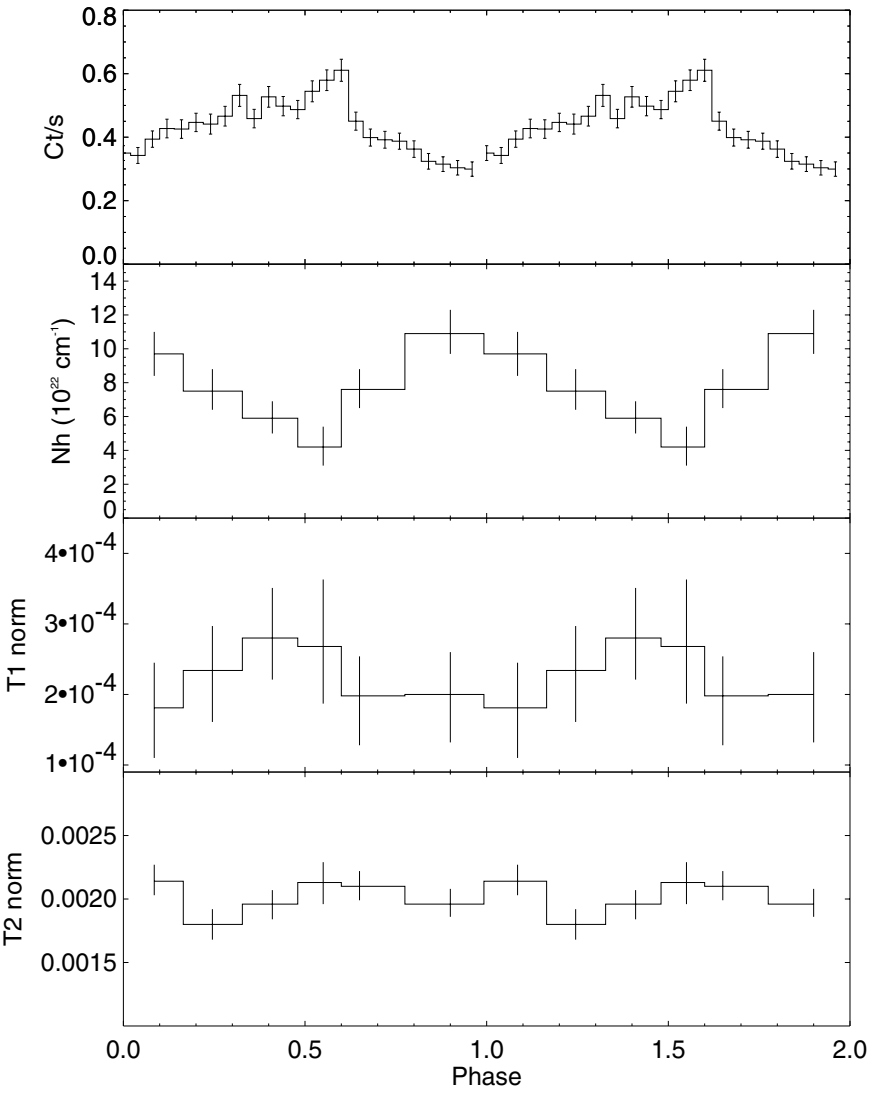

Fig. 4. From the top panel: using the time interval which showed evidence for a modulation on the $2193 \mathrm{~s}$ period, we fold that data on that period in the $0.15-1.0 \mathrm{keV}$ energy band; the fitted neutral absorption column; the normalisation of the lower temperature component $\left(k T_{1}\right)$; the normalisation of the higher temperature component $\left(k T_{2}\right)$.

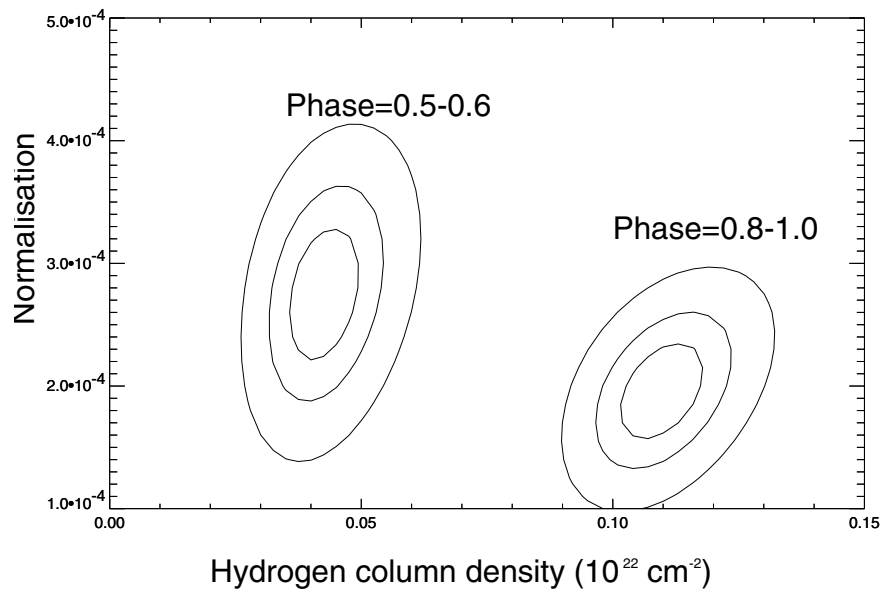

Fig. 5. Confidence contours in the $\left(N_{\mathrm{H}}, T_{1}\right.$, normalisation) plane for the phase minimum and maximum spectra. The contours correspond to $68 \%, 90 \%$ and $99 \%$ limits.

phase maximum and minimum: there is a clear separation in the contours.

We show in Fig. 6 the X-ray spectra near 6-7 keV for spectra taken from the phase maximum and minimum: the profile of the $\mathrm{Fe} \mathrm{K} \alpha$ line does not differ significantly. This further strengthens the argument that the spectral changes over

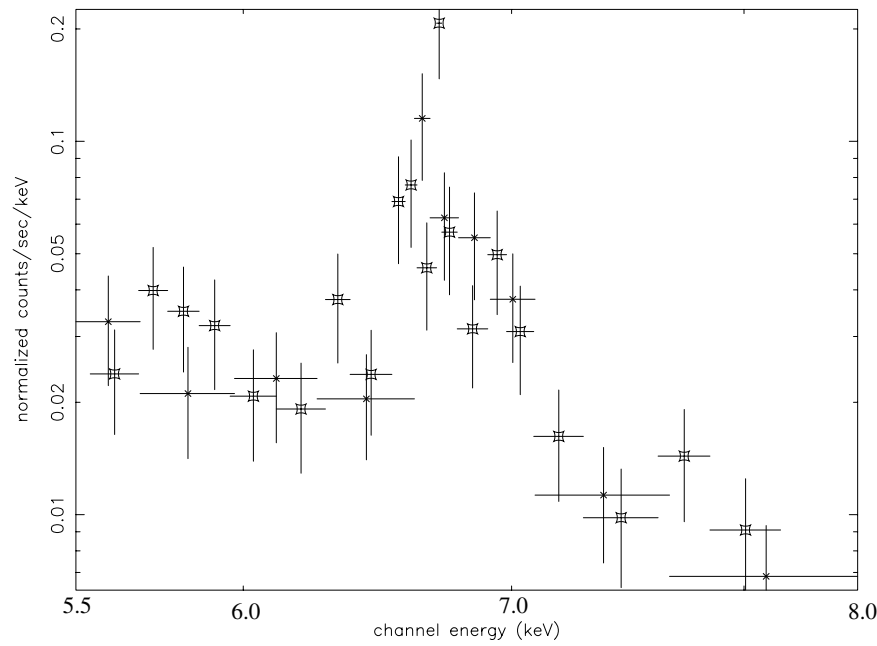

Fig. 6. The $\mathrm{Fe} \mathrm{K} \alpha$ complex at the 2193 s phase minima (0.5-0.6) (shown an asterix) and maxima (0.9-1.0) (shown as stars).

the 2193 s period are most likely caused by the change in the amount of absorption.

We also folded the data on the $3510 \mathrm{~s}$ quasi-stable modulation: we find a similar result to that found for folding on the 2193 s. Namely, the temperature and normalisation of the two thermal plasma components do not vary over phase, but the absorption component varies significantly. For brevity we do not show the results in table or figure form.

\section{Modelling the $B$ band and UV data}

It is clear from Ramsay et al. (2001a) that the $B$ and UV light curves exhibit clear modulation over the orbital period as well as asymmetric eclipse profiles. In this section we will describe and discuss the modelling of those data in detail.

Our basic assumption is that all of the $B$ and UV modulation is caused by the changing visibility of different parts of (at least) partially optically and physically thick accretion disc and the eclipse of such disc by the secondary star. In our modelling we have taken the idea of "fireflies" presented in Hakala et al. (2002) and developed it further to suit the modelling of optically thick accretion discs.

Hakala et al. (2002) use a swarm of emitting point sources (fireflies) to model the shape of an accretion stream in eclipsing AM Herculis systems. In their approach the swarm is regularized to favour realistic "banana" shapes for the accretion stream (without fixing the exact location or shape of the stream). They then used a variant of a genetic algorithm to find the optimal accretion stream shape and location.

If we want to apply a similar approach to locating the emitting regions in a disc accreting system, like OY Car, we need to make several modifications to the original code. In fact, the only assumptions that remain the same are the idea of "fireflies" as a numerical model for the emission and the optimisation algorithm. We next discuss our model computation in detail. 


\subsection{The model}

We first restrict the possible emission to originate within an accretion disc like volume around the primary. The boundary conditions for this volume are: maximum radius $0.45 a$, maximum height $\pm 0.02 a$. In addition we define a somewhat thicker disc like volume of maximum height $\pm 0.025 a$. This volume is assumed to have a homogenous optical thickness, that remains constant throughout the modelling. The reason for this is to provide estimates for the absorption from the different parts of the disc and thus guarantee an optically thick solution.

To compute the optical light curve for any given distribution of fireflies inside this boundary, the algorithm for the light curve generation procedes as follows. We first loop over all the orbital phases to be fitted and for each phase:

- select the fireflies that are not eclipsed by the secondary;

- determine the absorption coefficient for each of the selected fireflies. This is done by computing the length of a path that light from any particular fly has to travel inside a predefined disc volume;

- sum up the emission from all visible flies at this phase (taking into account the variable absorption for different flies).

The main difference between the "firefly approach" and the "normal approach" is that the firefly approach is (in analogue with hydrodynamics) a particle based solution whilst the "normal" approach relies on fixed grid on which the solution is determined. The reasons behind our choice of the firefly approach here are the following ones. Firstly, one of the main axioms of the standard eclipse mapping routine (Horne 1985) is that the eclipse profile of the disc is solely determined by the changing obscuration of the disc by the secondary. Thus outside the eclipse phases the light curve is constant. This implies that the same algorithm could not be used to fit the rest of the light curve. Normally the out-of-eclipse variation, often seen in quiescent state CV light curves, has to be removed before the fitting, which can cause undesirable bias in the results. Rutten (1998) developed the eclipse mapping by including a 3D shape for the disc. His model could be used for out-of-eclipse light curve modelling, but it has it's limitations as well, i.e. we have to know and fix the 3D shape of the disc beforehand.

The advantage of the firefly modelling is the lack of any grids. The flies are free to move within a predefined volume and their final distribution represents the location or distribution of emission within the disc. However, our approach does require that the disc has an axisymmetric homogenous density structure, as this is assumed for the computation of optical thickness effects that dominate the out-of-eclipse light curve shape.

\subsection{Model regularization}

As in most of inversion problems, there are an infinite number of solutions if no regularization is employed. In order to overcome this, we have (like many previous authors) decided to search for the most axisymmetric image that can reproduce the observations (axisymmetric about the accretion disc rotation axis). In the case of the firefly approach this simply means a regularization term, where we have defined the disc in $i$ sectors, and counted the number of flies in each sector $N_{i}$ (here we have chosen to use 36 sectors). Then we maximize the entropy of such division i.e. we first compute

$p_{i}=\frac{N_{i}}{\sum_{i=1}^{36} N_{i}}$

where $p_{i}$ is the fractional flux in each sector and the entropy term is:

$S=\sum_{i=1}^{36}-p_{i} \log \left(p_{i}\right)$

finally our merit function to be minimized is:

$F=\chi_{v}^{2}-\lambda S$

where $\chi_{v}^{2}$ is the standard reduced $\chi^{2}$ term, $\lambda$ is the Lagrangian multiplier and $S$ the entropy term.

\subsection{The fitting algorithm}

We carry out the actual fitting using the same genetic algorithm variant as in Hakala et al. (2002). The only difference is that in this case the initial population of solutions for fitting consists of disc like swarms of random fireflies. These are then evolved in similar fashion to fit the eclipse profile and the overall light curve simultaneously. As the whole light curve has 532 points in $B$ and 514 points in UV, we have decided to emphasize the eclipse phases $(0.95-1.05)$ by summing the Chi-square values from this phase range three times. We typically use 50 individual swarms in each generation and after around 1500 generations, the fits stop evolving in a reasonable time.

In order to test our approach we have created a synthetic light curve using a known disc brightness distribution, added appropriate levels of noise and fitted that. The results of this exercise are shown in Fig. 7. The top panel shows the whole orbital light curve together with the fit, the middle panel the phase range close to the eclipse phase and the bottom panel the synthetic brightness distribution (left) and the reconstructed distribution (right). The fits to the synthetic data are encouraging, with the reconstructed disc being close to the input disc. There is, however, a small amount of smearing, which is most likely caused by finite phase resolution, regularization and/or noise in the data.

\subsection{The results}

We have plotted in Figs. 8 and 9 the resulting brightness distribution from a fit to the $B$ and UV band OM data in a case where no regularization was applied. The fit is excellent, but the resulting brightness distribution suffers slightly from the "scissors" effects, i.e. the point spread function of the map has a scissors shape. This is because there is not enough information in the data to uniquely locate point like emission. Thus point source emission is spread out along the lines that mark the shadow of the secondary at the ingress and egress phases. This is a well known effect and was demonstrated by Horne (1985). 

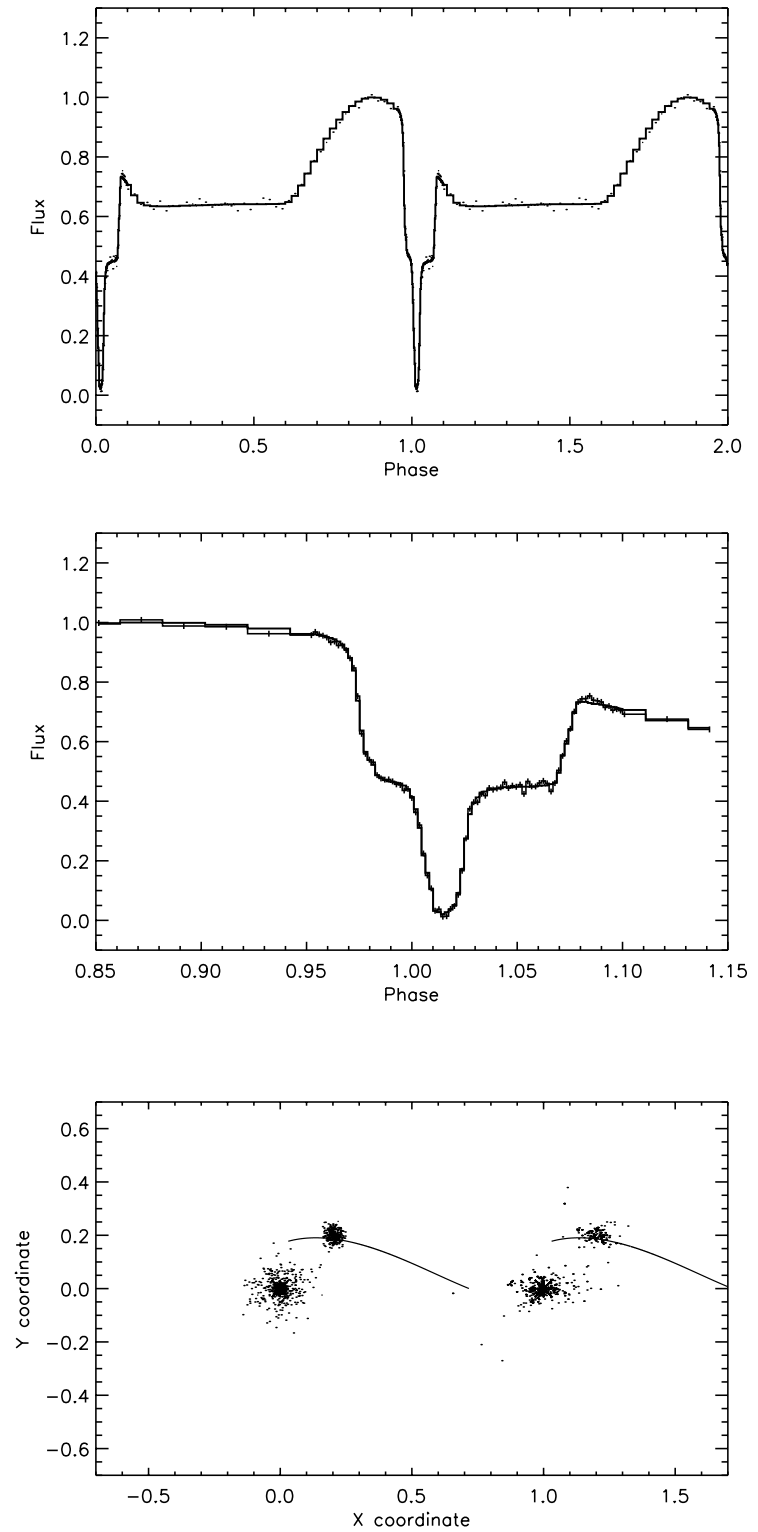

Fig. 7. Fits to the synthetic data. The whole light curve (top); zoomed in around the eclipse (middle); and the original (bottom, left) and reconstructed (bottom, right) brightness distributions for the fit. The bottom panel shows the ballistic stream trajectory and an accretion disc looking down on the orbital plane.

Using the regularization described earlier we obtain the fits for $B$ and UV bands shown in Figs. 10 and 11 respectively. The coordinate system for the fireflies is a Cartesian one, where the primary star is located in the origin and the $X-Y$ plane is the orbital plane. The coordinates of the $\mathrm{L} 1$ point are roughly $(0.7$, $0,0)$ and a ballistic freefall stream is overplotted. The observer lies "above" the orbital plane, i.e. she/he would have a positive Z-coordinate.

Figure 12 shows the radial histograms of the distribution of flies. The resulting brightness distributions seem to indicate that whilst the UV emission comes mostly from the white dwarf or the boundary layer and the inner disc, the optical $B$ band emission also contains a faint component from the accretion stream-disc interactions, i.e. the hot spot (and maybe
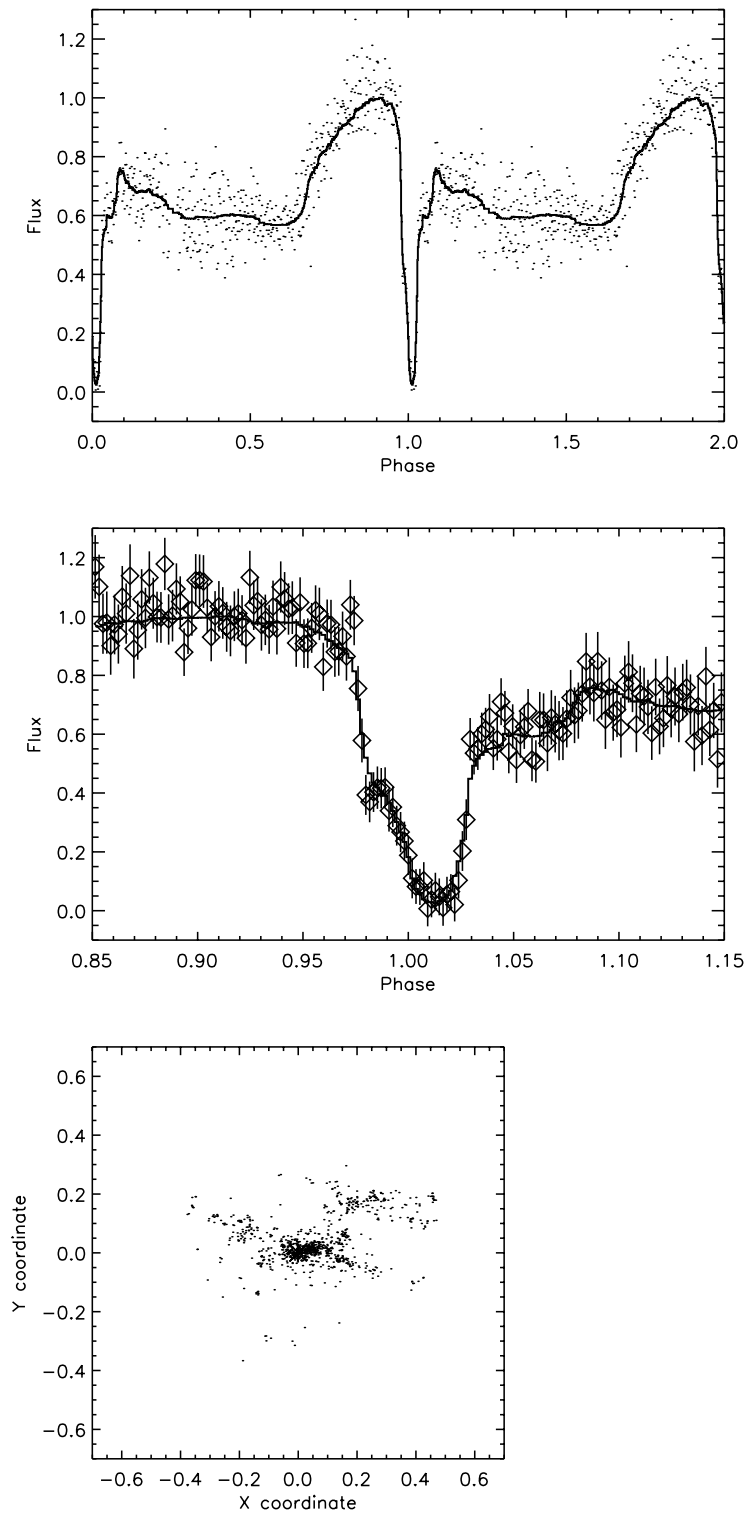

Fig. 8. Our best fit to the $B$-band data assuming no regularization. The whole light curve is shown on top and we have zoomed in around the eclipse (middle). The resulting firefly distribution together with a ballistic accretion stream trajectory is plotted in bottom (Primary at the origin, L1 point at approx. coordinates of $[0.7,0.0])$.

even from the freefall part of the stream). This is also rather obvious directly from the light curve shapes, given the difference in eclipse assymmetry between the two passbands.

Furthermore, we find that the hot spot is located exactly on the ballistic trajectory, as expected. The distance is $\sim 0.3 a$ from the white dwarf, which confirms the values reported by Wood et al. (1989). In addition to this we find marginal evidence for optical emission from the accretion stream prior to the main hot spot impact region. Our results show, that in the $B$ band $90 \%$ of the emission comes within a distance of $0.29 a$ from the white dwarf and $95 \%$ within $0.33 a$. This implies that the hot spot contributes roughly $5 \%$ of the total $B$ band emission. The same radii in UV band are slightly greater in comparison $(0.32 a$ and $0.38 a)$. At face value this implies that the $B$ band radial emission distribution is concentrated more towards the 

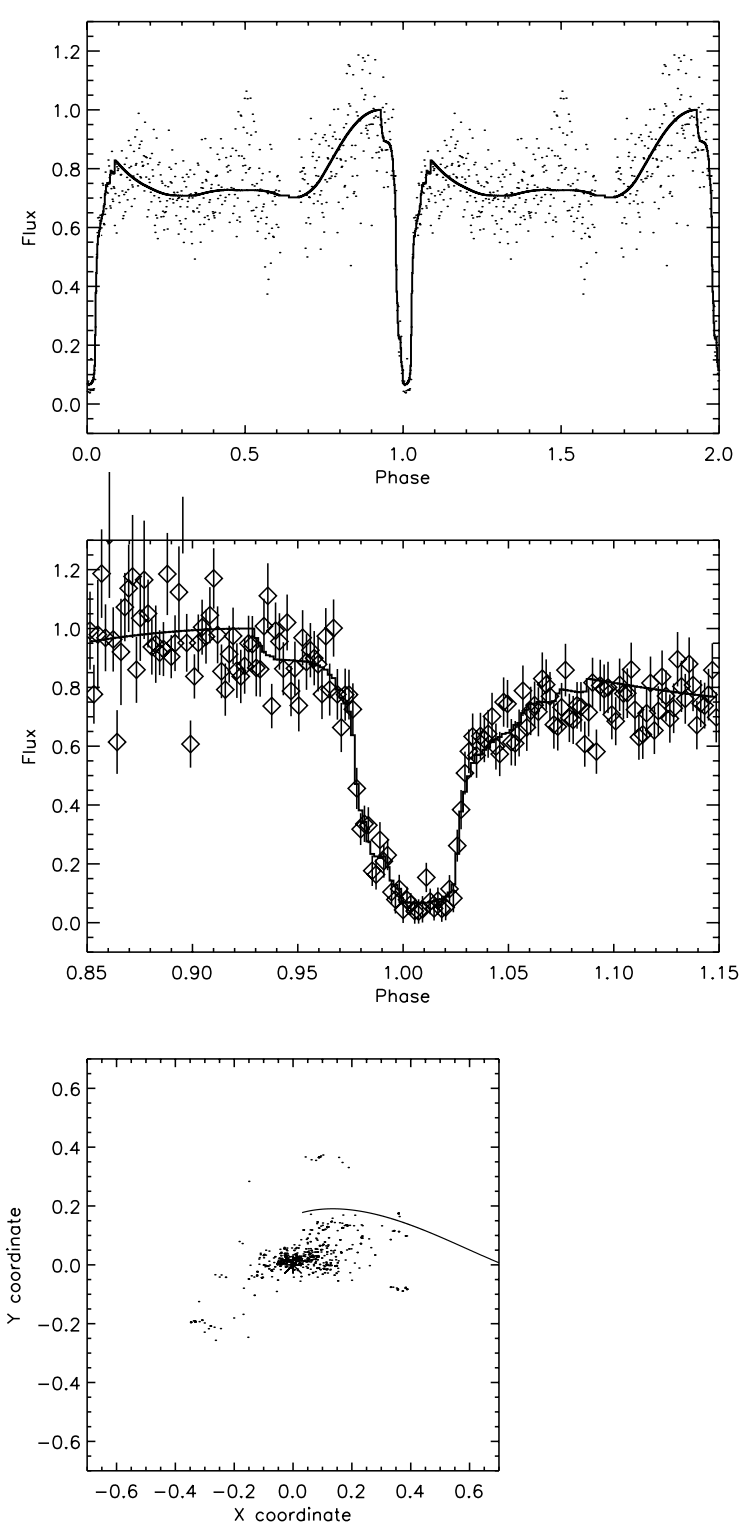

Fig. 9. Our best fit to the UV data assuming no regularization. The whole light curve is shown on top and we have zoomed in around the eclipse (middle). The resulting firefly distribution together with a ballistic accretion stream trajectory is plotted in bottom (Primary at the origin, L1 point at approx. coordinates of $[0.7,0.0]$ ).

white dwarf than in UV passband. This difference could be due to the fact that whilst our UV data was taken 4 days after an outburst, the $B$ band data was taken more than a month later. Dwarf novae discs are thought to be more uniformly bright during the outbursts. Although the $V$ band mag was similar in both observations this does not preclude a difference in the size of the accretion disc in the two observations.

\section{Discussion}

\subsection{The origin of the $X$-ray modulation}

Ramsay et al. (2001a) found that in X-rays the duration of the eclipse ingress and egress is $20-30 \mathrm{~s}$, which is roughly the same time as that expected for the secondary star to eclipse
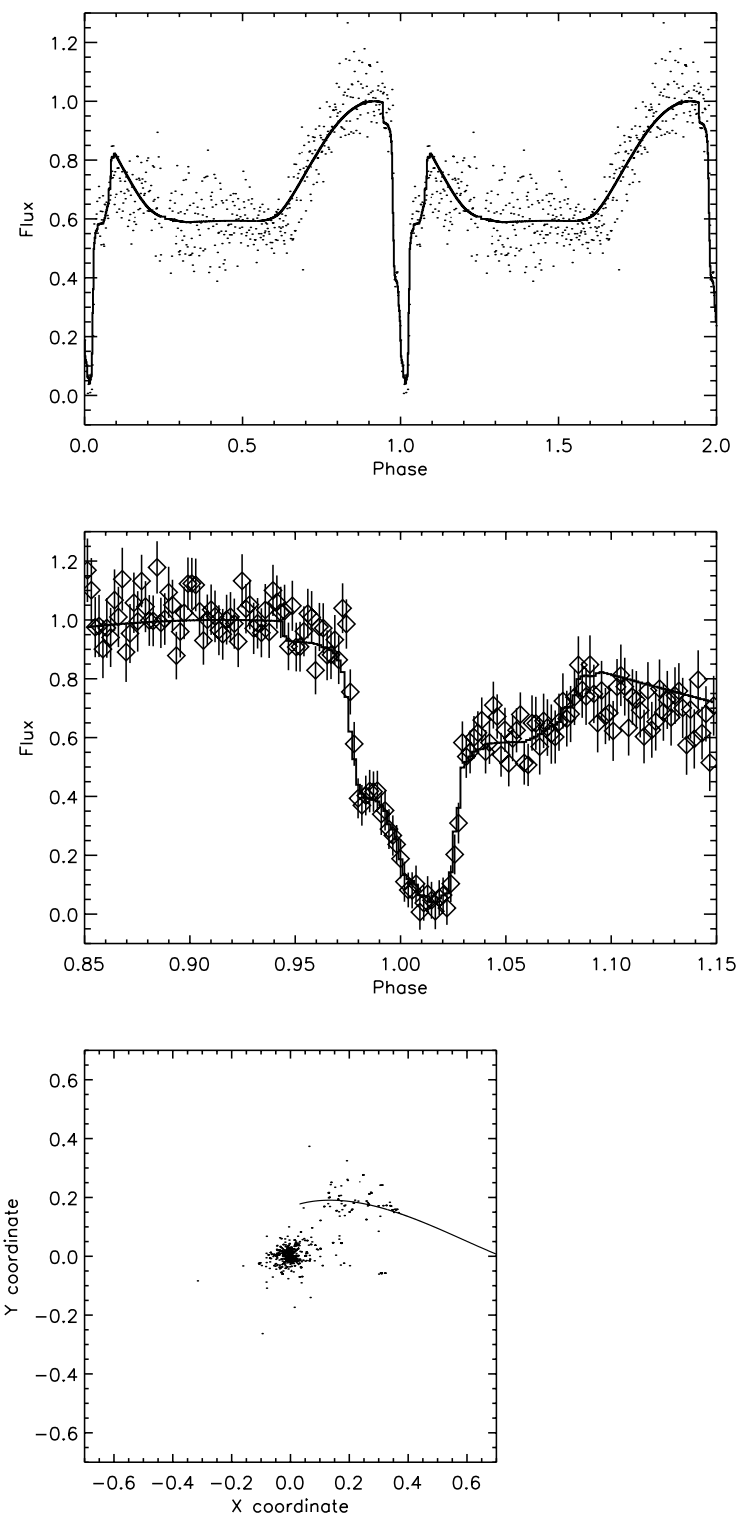

Fig. 10. Our best fit to the $B$-band data assuming axisymmetric regularization. The whole light curve is shown on top and we have zoomed in around the eclipse (middle). The resulting firefly distribution together with a ballistic accretion stream trajectory is plotted in bottom (Primary at the origin, L1 point at approx. coordinates of [0.7, 0.0]).

the diameter of the white dwarf. These authors concluded that the X-ray emission region is hence extended in white dwarf longitude, probably centered near the spin equator. However, Wheatley \& West (2003), using a re-analysis of the same data, conclude that the X-ray emission originated close to the upper pole on the white dwarf. In the latter scenario, an accretion column, or curtain, of material will rotate on the spin period of the white dwarf.

This is consistent by our spectral modelling in Sect. 4 which indicates that the most likely cause of these quasi-stable modulations is a variation in the absorption column over this period. If the $2193 \mathrm{~s}$ period was the spin period of the white dwarf, how would that manifest itself as a variation in the absorption column? In the case of intermediate polars, some systems show 

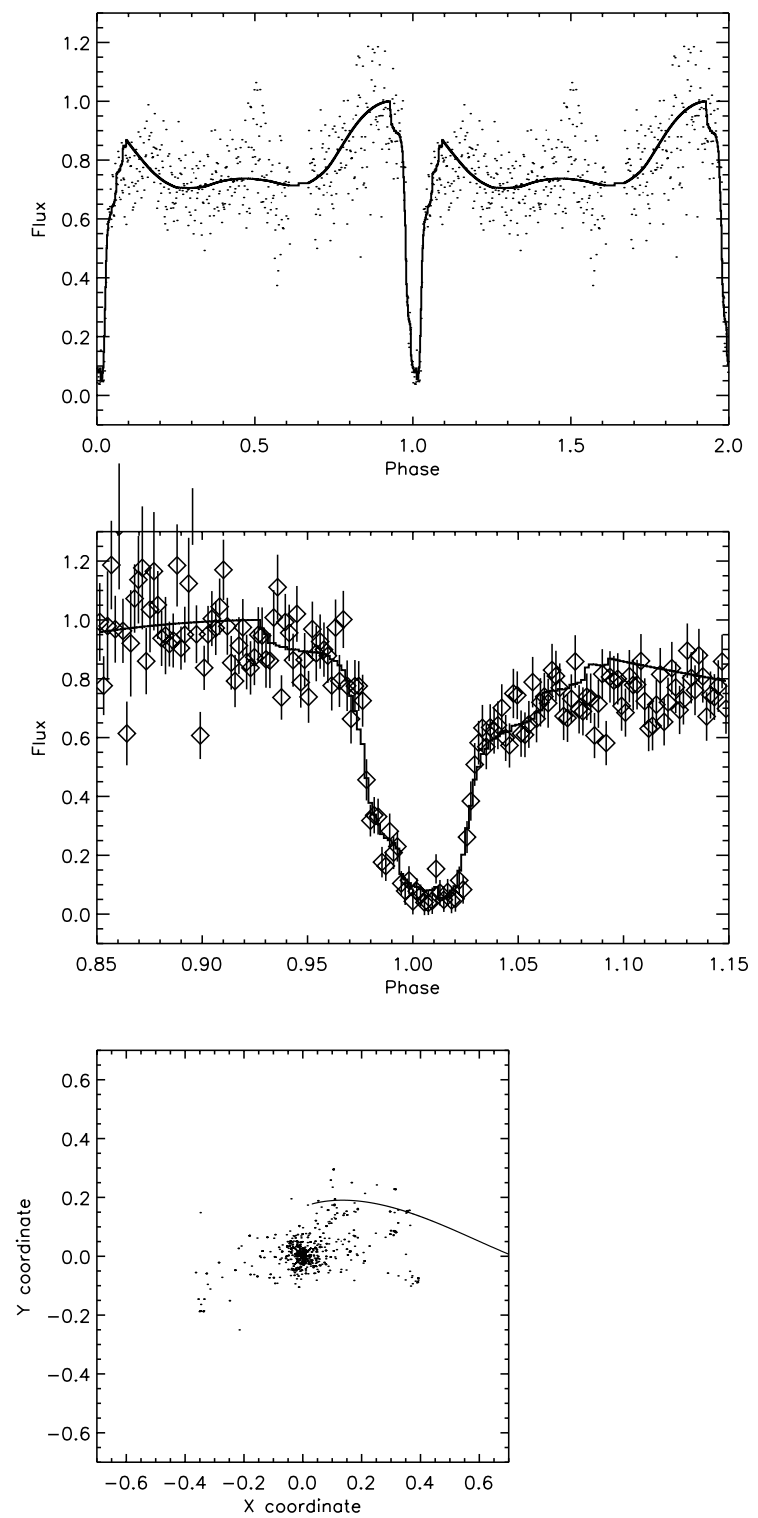

Fig. 11. Our best fit to the UV data (with axisymmetric regularization). The whole light curve is shown on top and we have zoomed in around the eclipse (middle). The resulting firefly distribution (bottom).

an energy dependent X-ray variation at the spin period. This has been seen in EX Hya (Rosen et al. 1988; Cropper et al. 2002) and is thought to be due to observing the emission site through an accretion curtain whose absorption column varies as the white dwarf rotates.

A second possibility may be due to a variation in the distance from the inner edge of the accretion disc to the white dwarf. Further, this distance could vary in azimuth in the binary frame. Thus the white dwarf-accretion disc interaction would lead to a variation at a beat period and could account for why the modulation is only seen for around $1 / 3$ of the observation duration. This hypothesis requires that the $2193 \mathrm{~s}$ period is the spin period of the white dwarf $(\omega)$, whilst the 3510 s modulation is the beat period between the spin and the $5436 \mathrm{~s}$ orbital period $(\Omega)$. The beat period based on the $2193 \mathrm{~s}$ spin period would be $(\omega-\Omega) 3676 \mathrm{~s}$, which is $6.6 \sigma$ distant from the best

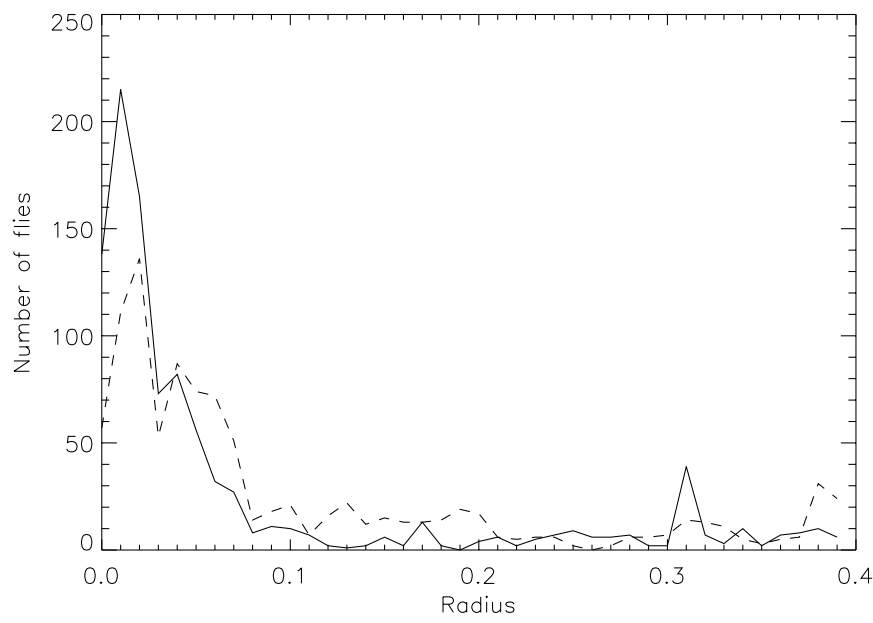

Fig. 12. The radial distribution of fireflies in $B$ (solid) and UV bands (dashed). The hot spot in $B$ band is visible at a distance of $0.3 a$.

fit period. However, if the spin period is actually $1.7 \sigma$ shorter than the best fit value (giving $2133 \mathrm{~s}$ ) then the observed $3510 \mathrm{~s}$ period would be exactly the expected $\omega-\Omega$ beat period. In this scenario, any very short periods (for instance the 17.94 and 18.15 s periods reported by Marsh \& Horne 1998) would have to be explained in other terms, for instance white dwarf oscillations.

Finally, Marsh \& Horne (1998) suggested that one of the $\sim 18$ s periods could represent $\omega$, or alternatively that one of the periods was the Keplerian period and the other was the beat period between this and the spin period (implying a spin period of $\sim 1550 \mathrm{~s}$ ). If $\sim 1550 \mathrm{~s}$ was the spin period, then most of the amplitude peaks in our Fig. 1 could be explained as naturally occuring sidebands of the spin and orbital frequencies in the following manner: $1248 \mathrm{~s}(\omega+\Omega), 1574 \mathrm{~s}(\omega), 2193 \mathrm{~s}$ $(\omega-\Omega), 4844 \mathrm{~s}(2(\omega-\Omega))$. The main drawback with this hypothesis is that there is no simple explanation for the very significant $3510 \mathrm{~s}$ period. Also, it is possible that not all of the 5 periods mentioned above are in fact physical (cf. Sect. 3 ).

Recently, the short period variability in cataclysmic variables has been extensively discussed in a series of papers by Warner \& Woudt (most recently in Warner et al. 2003). In conclusion they have found that typically $P_{\mathrm{QPO}} \sim 15 P_{\mathrm{DNO}}$. In addition they also find second type of DNO's with: $P_{\mathrm{lpDNO}} \sim$ $4 P_{\mathrm{DNO}}$. Assuming that one of the periods seen here, $2193 \mathrm{~s}$ or $3500 \mathrm{~s}$, would be a QPO period the above mentioned relationship would then predict DNO periods around $150 \mathrm{~s}$ and $230 \mathrm{~s}$ respectively. There is no evidence for such periods in our data. However, this is expected since the DNO and QPO periods are normally found during outbursts, and during our observation the source was in quiescence.

We conclude that based on our data, we cannot provide a unique explanation for the $2193 \mathrm{~s}$ and $3510 \mathrm{~s}$ periods. We can, however, conclude that these periods occur due to changes in the line of sight absorbing column. 


\subsection{The optical light curve modelling}

Much previous work has been done on the eclipse profiles of OY Car ranging from infrared to X-rays (see for instance Berriman 1984, 1987; Cook 1985; Krzeminski \& Vogt 1985; Schoembs 1986; Wood et al. 1989; Rutten et al. 1992; Horne et al. 1994; Bruch et al. 1996; Pratt et al. 1999; Ramsay et al. 2001a; Wheatley \& West 2003). Wood et al. (1989) use the optical eclipse profiles to measure accurate system parameters and assuming a ballistic free fall trajectory for the accretion stream they deduce a disc outer radius of approximately $0.31 a$. Rutten et al. (1992) re-analysed the set of eclipse profiles from Vogt (1983) that cover the rise into an outburst. Their eclipse mapping analysis shows that whilst the quiescence eclipse profiles are dominated by the white dwarf and the hot spot, the rest of the disc becomes dominant in the outburst. The HST UV observations by Horne et al. (1994) demonstrate that the eclipse profiles become more symmetric and "tophat shaped" towards the shorter wavelengths. This indicates that the white dwarf and/or the boundary layer is the major source of emission in the UV, whilst the hot spot contribution rises towards the optical wavelengths.

Our light curve modelling confirms the previous results on the origin of the quiescent eclipse profiles. In particular they indicate, that both in $B$ and UV bands, the majority of the emission arises close to the white dwarf, possibly either the boundary layer or the white dwarf itself. This is also supported by the ingress and egress time analysis of the the same $B$, UV dataset by Ramsay et al. (2001a).

The freefall accretion stream and especially the hot spot, where the stream hits the disc outer edge is mainly emitting in the $B$ band and hardly visible in the UV maps. Our modelling also suggests that in addition to the hot spot, the ballistic accretion stream might contribute to the $B$ band emission as well.

Our novel approach allows simultaneous fitting of the eclipse profiles and orbital light curves of cataclysmic variables. This removes bias introduced by artificially having to remove the orbital modulation prior to the eclipse profile mapping. Furthermore, the "firefly approach" has potential to be easily converted into full 3D mapping, if a proper regularisation can be employed.

\section{Conclusions}

We have reanalysed the XMM-Newton observations of the dwarf novae OY Car. In particular, we have reanalysed the $\mathrm{X}$-ray light curve and spectrum and developed a new model to fit the OM $B$ band and UV data.

We have investigated the X-ray light curve over the time interval where the previously reported $2240 \mathrm{~s}$ modulation was strongly seen. This has allowed us to refine this period to $2193 \mathrm{~s}$. We also detect a modulation at a period of $3510 \mathrm{~s}$ which we also believe to be significant. We have performed phase resolved X-ray spectroscopy on these periods and find that the soft X-ray light curve is anti-correlated with the absorption column density. This implies that the light curve modulation is due to us observing the $\mathrm{X}$-ray emission region through a rotating accretion column or curtain. Although, not conclusive, this supports the hypothesis of Ramsay et al. (2001a) that the $2193 \mathrm{~s}$ is the spin period of the white dwarf. Furthermore, this is in agreement with findings of Wheatley \& West (2003), where they conclude that the X-ray emission probably originates near the polar region on the white dwarf surface.

To model the OM $B$ and UV light curves, we have applied the fireflies model of Hakala et al. (2002). We simulated light curves using a given accretion disc brightness distribution and used our technique to fit the synthetic light curves. The resulting distribution of fireflies is encouraging. We then applied the model to the OM data and find that the hot-spot, where the accretion stream hits the disc, is exactly on the ballistic trajectory. Further, we find some evidence that the $B$ band emission is more concentrated than the UV emission, although this maybe due to the fact that the $B$ band data were taken 1 month after the UV data. Our technique can be applied to other disc accreting systems.

Acknowledgements. This paper is based on observations obtained with XMM-Newton, an ESA science mission with instruments and contributions directly funded by ESA Member States and the USA (NASA). PJH is an Academy of Finland research fellow.

\section{References}

Aschenbach, B., Briel, U., Haberl, F., et al. 2000, SPIE 4012, Paper 86 [astro-ph/0007256]

Berriman, G. 1984, MNRAS, 207, 783

Berriman, G. 1987, MNRAS, 228, 729

Bruch, A., Beele, D., \& Baptista, R. 1996, A\&A, 306, 151

Cash, W. 1979, ApJ, 228, 939

Cook, M. C. 1985, MNRAS, 215, 211

Cropper, M., Ramsay, G., Hellier, C., et al. 2002, Phil. Trans. R. Soc. Lond. A., 360, 1951

Hakala, P. J., Cropper, M., \& Ramsay, G. 2002, MNRAS, 334, 990

Horne, K. 1985, MNRAS, 213, 129

Horne, K., Marsh, T. R., Cheng, F. H., Hubeny, I., \& Lanz, T. 1994, ApJ, 426, 294

Krzeminski, W., \& Vogt, N. 1985, A\&A, 144, 124

Mason, K. O., Breeveld, A., Much, R., et al. 2001, A\&A, 365, L36

Marsh, T., \& Horne, K. 1998, MNRAS, 299, 921

Pratt, G. W., Hassall, B. J. M., Naylor, T., Wood, J., \& Patterson, J. 1999, MNRAS, 309, 847

Ramsay, G., Poole, T., Mason, K., et al. 2001a, A\&A, 365, L288

Ramsay, G., Córdova, F., Cottam, J., et al. 2001b, A\&A, 365, L294

Rosen, S. R., Mason, K. O., \& Córdova, F. 1988, MNRAS, 231, 549

Rutten, R. 1998, A\&AS, 127, 581

Rutten, R. G. M., Kuulkers, E., Vogt, N., \& van Paradijs, J. 1992, A\&A, 265, 159

Schoembs, R. 1986, A\&A, 158, 233

Strüder, L., Briel, U., Dennerl, K., et al. 2001, A\&A, 365, L18

Vogt, N. 1983, A\&A, 128, 29

Warner, B., Woudt, P. A., \& Pretorius, M. L. 2003, MNRAS, 344, 1193

Wheatley, P. J., \& West, R. G. 2003, MNRAS, 345, 1009

Wood, J. H., Horne, K., Berriman, G., \& Wade, R. A. 1989, ApJ, 341, 974 